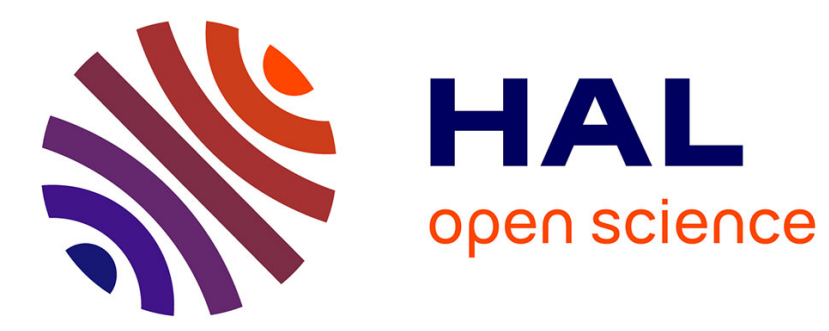

\title{
To the theory of ferrohydrodynamic circulating flow induced by running magnetic field
}

Andrey Yu Zubarev, Maxim Raboisson-Michel, Gregory Verger-Dubois, Pavel Kuzhir

\section{- To cite this version:}

Andrey Yu Zubarev, Maxim Raboisson-Michel, Gregory Verger-Dubois, Pavel Kuzhir. To the theory of ferrohydrodynamic circulating flow induced by running magnetic field. The European Physical Journal. Special Topics, 2020, 229 (19-20), pp.2961-2966. 10.1140/epjst/e2020-000119-3 . hal-03088289

\section{HAL Id: hal-03088289 \\ https://hal.science/hal-03088289}

Submitted on 25 Dec 2020

HAL is a multi-disciplinary open access archive for the deposit and dissemination of scientific research documents, whether they are published or not. The documents may come from teaching and research institutions in France or abroad, or from public or private research centers.
L'archive ouverte pluridisciplinaire HAL, est destinée au dépôt et à la diffusion de documents scientifiques de niveau recherche, publiés ou non, émanant des établissements d'enseignement et de recherche français ou étrangers, des laboratoires publics ou privés. 


\title{
To the theory of ferrohydrodynamic circulating flow induced by running magnetic field.
}

\author{
Andrey Yu. Zubarev ${ }^{1,2}$ Maxim Raboisson-Michel ${ }^{3,4}$, Gregory Verger-Dubois ${ }^{4}$ and \\ Pavel Kuzhir ${ }^{3}$
}

${ }^{1}$ Theoretical and Mathematical Physics Department, Institute of Natural Sciences and Mathematics, Ural Federal University, Lenin Ave, 51, Ekaterinburg, 620083, Russia,

${ }^{2}$ M.N. Mikheev Institute of Metal Physics of the Ural Branch of the Russian Academy of Sciences, Ekaterinburg, Russia

${ }^{3}$ Université Côte d'Azur, CNRS UMR 7010, Institute of Physics of Nice, Parc Valrose, 06108 Nice, France

${ }^{4}$ Axlepios Biomedical - lere Avenue 5eme rue, 06510 Carros, France

\begin{abstract}
We present results of theoretical modelling of macroscopic circulating flow induced in a drop of ferrofluid by oscillating running magnetic field. The drop is placed in a narrow flat channel filled by a non-magnetic liquid. The aim of this work is development of a scientific basis for a progressive method of address drug delivery to thrombus clots in blood vessels with the help of the magnetically induced circulation flow. Our results show that the oscillating running field allows inducing the carrier fluid flow with velocity amplitude $1-10 \mathrm{~cm} / \mathrm{s}$. This is the range of values, presenting interest from the point of view of the drug delivery.
\end{abstract}

Keywords: Magnetic fluid; alternating magnetic field; field-induced flow.

\section{Introduction}

Most common method of the treatment of brain stokes consists in injection of tissue plasminogen activator to dissolve the blood clots and restore the blood flux. However, the thrombolytic drug diffuses very slowly toward blood clots through blocked vessels. A smart solution of this problem has been proposed in [1]. The key idea of this method is injection of a ferrofluid with nano-sized magnetic particles in the blocked vessel and generation of recirculating flows under applied external magnetic field. Under the action of the applied field, the magnetic nanoparticles or their needle-shaped aggregates become to rotate; this rotation provokes recirculating flows in the blocked vessels which in turn considerably enhance convective transport of the drug towards the clots. Only a few works have been published on this topic [2-4]. However, the physical understanding of the origin of the recirculating or oscillatory flows is necessary for development and practical application of this method.

A theoretical analysis of the circulation flow in a liquid in a flat channel, a few moments after injection of a ferrofluid drop, under the action of a rotating field has been proposed in ref.[5]. The considered ferrofluid was supposed to consist of individual spherical particles with permanent magnetic moments. It was shown that the flow appears only under the field with spatially nonuniform amplitude and only in the presence of nanoparticle concentration gradients (naturally induced by injection of a ferrofluid drop). Possible nanoparticle aggregation induced by applied magnetic fields observed in experiments and considerably enhancing recirculation flows $[3,4]$ was not taken into account. 
In this paper, we propose a modified theoretical model of the circulation flow induced in a drop of magnetic fluid, situated in a thin channel, modelling a blood vessel. The ferrofluid consists of elongated magnetizable particles mimicking field induced aggregates. Furthermore, we consider not rotating, but oscillating running magnetic fields. They can be generated using only one pair of Helmholtz coils and would allow a much more compact design of the final medical device than the rotating magnetic fields requiring two pairs of Helmholtz coils or mechanically rotating permanent magnets [1]. The obtained results demonstrate that the velocity of the field-induced recirculating flows in a closed channel is well above the minimal value required for efficient drug delivery in real blood vessels. This is quite encouraging result, and we believe that the brain stroke treatment can be realized with low magnetic fields/ electric currents, while the developed theoretical model could be useful for parametric optimization of this medical technique.

\section{Mathematical model}

We consider a flat channel of an infinite size in the $y$ direction and with two opposite solenoids placed at the extremities along the horizontal axis $x$, as illustrated in Fig.1.

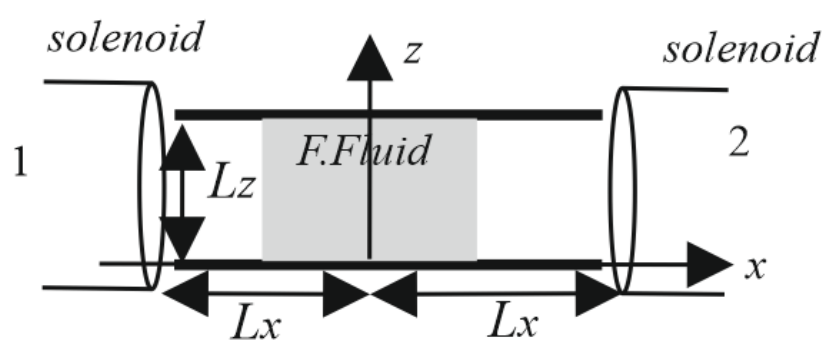

Fig.1. Sketch of the flat channel with a ferrofluid drop (gray rectangle) injected in the middle of the channel.

The distance $2 L_{x}$ between the solenoids is supposed to be much larger than the channel thickness $L_{z}$. For simplicity, we suppose that inside the ferrofluid drop the viscosity $\eta$ is homogeneous and is the same as outside the drop. This means that at any moment of time, the volume concentration $\Phi$ of the particles inside the drop is relatively low, about a few volume per cent. A drop of the ferrofluid is situated in the middle, between the solenoids. The initial volume concentration $\Phi_{0}$ of the particles is supposed to be known and depends only on the coordinate $x$. For the sake of simplicity, we consider here the two-dimensional model of the system, and suppose that all physical events take place in the plane $x, z$. Then, we consider the linear dependence of the ferrofluid magnetization $\mathbf{M}$ on the local magnetic field. Note that this approximation is not principal and cannot affect qualitative effects revealed by the present model. The "non-linear" generalization will make the calculations more cumbersome.

We suppose that magnetic fields $\mathbf{H}_{1}$ and $\mathbf{H}_{2}$, created by the solenoids 1 and 2, take the form:

$H_{1 x}=h_{1 x}(x, z) \cos \omega t ; \quad H_{1 z}=h_{1 z}(x, z) \cos \omega t$

$H_{2 x}=h_{2 x}(x, z) \sin \omega t ; \quad H_{2 z}=h_{2 z}(x, z) \sin \omega t$

where $\omega$ is the angular frequency of the alternating magnetic field, $t$ is the time and the spatial parts $\boldsymbol{h}(x, z)$ of the fields can be calculated by using the standard formulas for the fields of the cylindrical solenoids with given values of the current intensity, radius and length. Exact 
expressions for $h(x, z)$ are provided in our previous work [5]. Below we will use the notation $T=$ $2 \pi / \omega$ for the period of the field oscillation The results of calculations of the local absolute value $H(x, z, t)$ of the total field $\mathbf{H}=\mathbf{H}_{1}+\mathbf{H}_{2}$ are illustrated in Fig. 2 for the realistic solenoid parameters indicated in the figure caption.
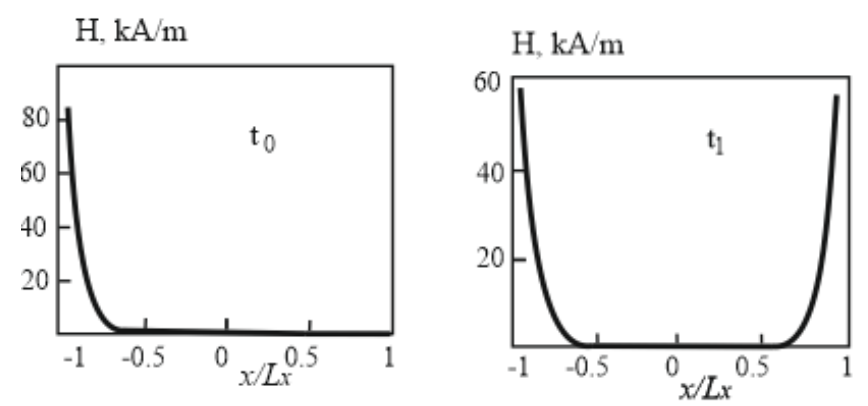

$\mathrm{H}, \mathrm{kA} / \mathrm{m}$
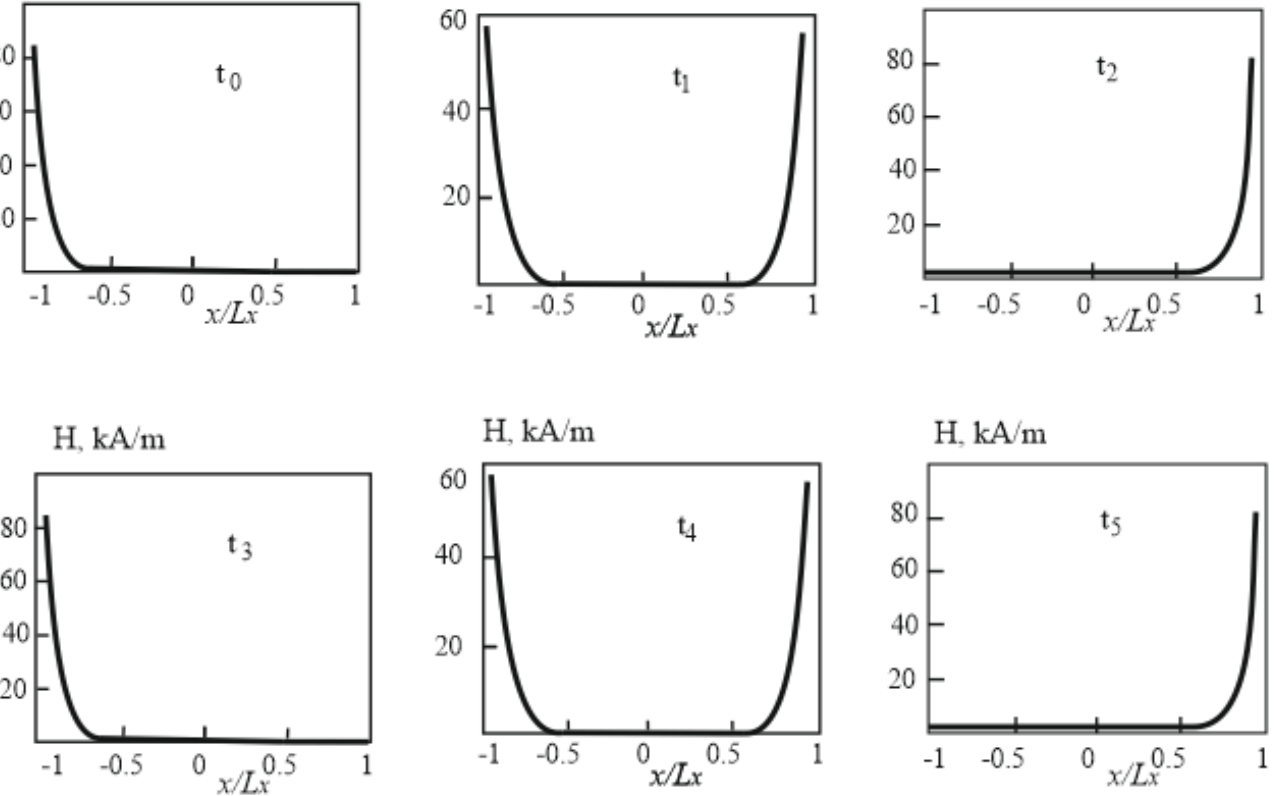

Fig.2. Absolute value $H$ of the instantaneous local field $\mathbf{H}$ vs. the horizontal coordinate $x$ for $z=L_{z} / 2$ (middle of the channel thickness) for the time moments: $t_{0}=k T / 2 ; t_{1}=k T / 2+T / 8 ; t_{2}=k T / 2+T / 4$; $k=0,1,2, \ldots$ The solenoid parameters are as follows: radius $10 \mathrm{~cm}$; length $5 \mathrm{~cm}$; the product $I_{0} N=$ $18 \mathrm{kA}$, where $I_{0}$ is current amplitude and $N$ is the number of turns; the current angular frequency $\omega=15 \mathrm{~s}^{-1}$. The distance between the solenoids $2 L_{x}=20 \mathrm{~cm}$; the channel thickness $L_{z}=5 \mathrm{~mm}$

We suppose that the ferrofluid consists of highly elongated magnetizable particles (mimicking field-induced aggregates composed of spherical ferrofluid nanoparticles). At this stage, we ignore the possible aggregation of the elongated particles as it was not reported in experimental studies $[3,4]$.Estimates show that the time of the particle reorientation in the alternating field is much smaller than the period of the field oscillation with the typical angular frequency $\omega \sim 10-20 \mathrm{~s}^{-1}$ We can therefore suppose that the elongated particles are always oriented along the local magnetic field $\mathbf{H}$. At such condition, the torque term (proportional to $\mathbf{M} \times \mathbf{H}$ ) disappears from the equation of ferrofluid motion. One can also show that for the considered channel dimensions and for oscillating flows with the typical frequency $\omega \sim 10-20 s^{-1}$, the inertia terms can also be ruled out from the equation of motion. Under these approximations, equations of motion of the whole ferrofluid and of its solid phase (composed of elongated particles) take the following form [6]:

$\mathbf{0}=-\nabla p+\eta \Delta \boldsymbol{v}+\Phi \boldsymbol{F}_{m}$

$\operatorname{div} \boldsymbol{v}=\mathbf{0}$

$\frac{\partial \Phi}{\partial t}+\operatorname{div}\left(\Phi \boldsymbol{F}_{m} \frac{V_{a}}{\beta}\right)+\operatorname{div}(\Phi \boldsymbol{v})=\mathbf{0}$ 
Here $v$ is the flow velocity, $p$ is the pressure, $\Phi(x, z, t)$ is volume concentration of the particles, $\boldsymbol{F}_{m}$ is the magnetic force acting by unit volume of each elongated particle by the magnetic field gradient, $V_{a}$ is the particle volume, $1 / \beta$ is the component of the hydrodynamic mobility tensor of the elongated particle along the force $\boldsymbol{F}_{m}$.

In the frames of the linear magnetization approximation, the magnetic force by unit volume of elongated particles can be estimated as follows: [7]

$F_{m}=-\nabla U, \quad U=-\frac{\mu_{0}}{2} \chi_{a} H^{2}$

Here $U$ is the potential energy of elongated particles oriented at each point along the local the field $\boldsymbol{H}(x, z, t)$ and evaluated under realistic approximation of high length-to-diameter ratio of particles, $\chi_{a}$ is magnetic susceptibility of these particles, and $\mu_{0}=4 \pi \times 10^{-7} \mathrm{H} / \mathrm{m}$ is magnetic permeability of vacuum. .

In the frames of these approximations, applying rot operator to the both parts of the first equation (1a), one gets:

$\Delta \operatorname{rot} v=\frac{1}{\eta} \operatorname{rot}(\Phi \nabla U)$

This is convenient to introduce the standard stream function $\Psi$ :

$v_{x}=\frac{\partial \Psi}{\partial z}, \quad v_{z}=-\frac{\partial \Psi}{\partial x}$

By using (3), (4) in (2), we come to the following relation:

$\Delta^{2} \Psi=-\frac{1}{\eta}\left[\frac{\partial U}{\partial z} \frac{\partial \Phi}{\partial x}-\frac{\partial U}{\partial x} \frac{\partial \Phi}{\partial z}\right]$

The boundary conditions for the function $\Psi$ are:

$\Psi=0$, at $z=0, L_{z}$

$\frac{\partial \Psi}{\partial z}=0$, at $z=0, L_{z}$

$\Psi \rightarrow 0$, at $x \rightarrow \pm \infty$

We should solve eq. (5), (6) together with the third equation of (1). In can be done only numerically.

To get some transparent analytical results, let us consider the initial stage of the oscillations, a few moments after the injection of a ferrofluid droplet, when the concentration $\Phi$ is practically equal to its initial profile $\Phi_{0}(x)$.

Since $L_{z}<<L_{x}$, all derivates of $\Psi$ over $z$ are much larger than the derivates over $x$. Taking it into account, the equation (5) reduces to:

$\frac{\partial^{4}}{\partial z^{4}} \Psi=-\frac{1}{\eta} \frac{\partial U}{\partial z} \frac{\partial \Phi_{0}(x)}{\partial x}$

Solution of the problem (4), (7) is:

$\Psi=-\frac{1}{\eta} \frac{\partial \Phi_{0}(x)}{\partial x} \int_{0}^{z} d z^{\prime} \int_{0}^{z \prime} d z^{\prime \prime} \int_{0}^{Z \prime \prime} U\left(x, z^{\prime \prime \prime}, t\right) d z^{\prime \prime \prime}+\frac{1}{6} A z^{3}+\frac{1}{2} B z^{2}$ 
where the integration constants $A, B$ are determined from the boundary condition $\Psi=0, \frac{\partial}{\partial z} \Psi=$ 0 at $z=L_{z}$. We omit their explicit expressions for brevity. Thus the calculation steps can be summarized as follows. Using a known concentration profile $\Phi_{0}(x)$, we first calculate the stream function with the help of Eq. (8). Then, replacing Eq. (8) in Eq. (4), we obtain both components $v_{x}$ and $v_{z}$ of the velocity profile. The numerical results allow us to compare the obtained velocity values with those required for successful treatment of brain strokes by the considered method.

\section{Results and discussion}

The calculations of the velocity profile in the flat channel were done for a gaussian-like profile of initial concentration, rather typical for fluid injection processes:

$\Phi_{0}(x)=\Phi^{0} \exp \left(-\frac{x^{2}}{\sigma^{2}}\right), \Phi^{0}=0.05 ; \sigma=1 \mathrm{~cm}$

This means that we consider a cloud of ferroparticles with the typical length $1 \mathrm{~cm}$. Another important parameter intervening into calculations is the magnetic susceptibility $\chi_{a}$ of these particles. We take the value $\chi_{a}=25$, close to the one $\left(\chi_{a}=24\right)$ reported in experiments with ferrofluid microdroplets [8]. Some results of calculations of the longitudinal component of the velocity $v_{x}=\frac{\partial \Psi}{\partial z}$ in the middle plane of the channel $\left(z=L_{z} / 2\right)$ are shown in Fig.3.
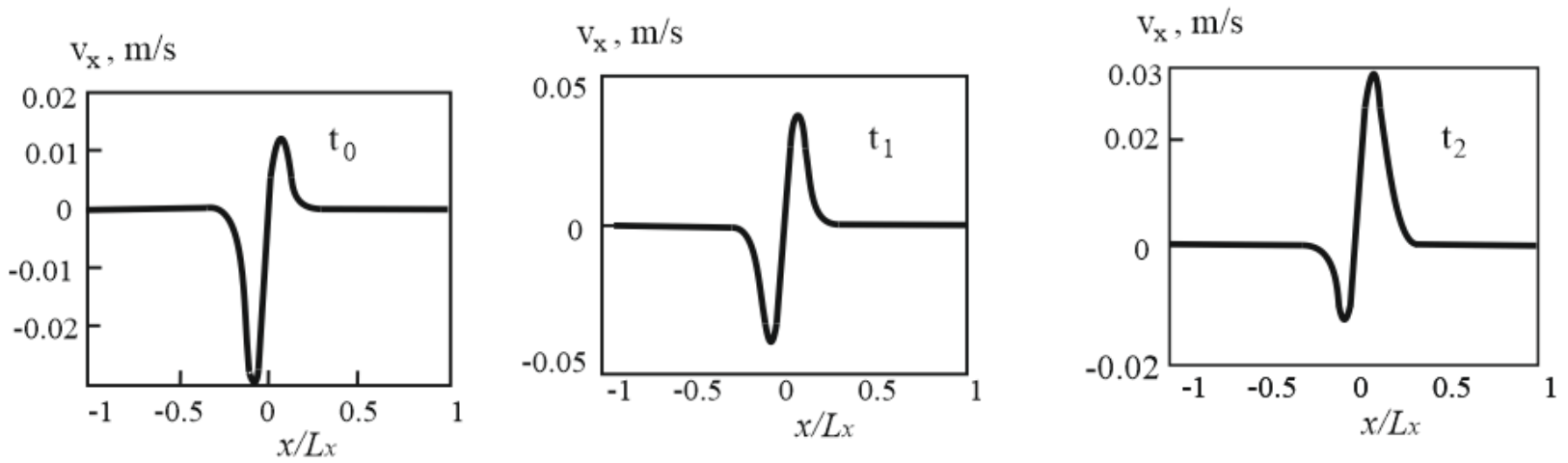

Fig.3. The longitudinal velocity $v_{x}$ at the middle plane of the flat channel vs. coordinate $x$ for several moments of time $t_{0}=k T / 2 ; t_{1}=k T / 2+T / 8 ; t_{2}=k T / 2+T / 4 ; k=0,1,2, \ldots$. The other parameters of the system are described in the figure caption of Fig. 2 .

The results show that for the chosen parameters of the system the velocity, close to the drop edges, can achieve several centimeters per second. The characteristic value $\left(\sim 10^{-3}-10^{-2} \mathrm{~m} / \mathrm{s}\right)$ obtained in our simulations is three-to-four orders of magnitude larger than the value $v_{x} \approx 3 \times 10^{-6} \mathrm{~m} / \mathrm{s}$ reported in experiments of [9] with fat ablation by generated microcirculation flows of magnetic suspension. Nevertheless, we warrant the reader that theoretical velocity values obtained in the present paper are valid only at the very onset after the magnetic field application, and the temporal evolution of the speed caused by spreading of the ferrofluid cloud along the channel has to be checked in order to confirm the effectiveness of the proposed method during a few minutes - a typical timescale for the medical intervention previewed for the brain stroke treatment by this method [1]. 


\section{Conclusion.}

Our results show that the running oscillating field with a quite realistic amplitude can induce in a ferrofluid drop, therefore in a liquid, the drop is situated in, circulating flows with the velocity amplitude about several centimeters per second. This effect can be used for the intensification of drug delivery in blood vessels.

\section{Acknowledgements}

PK acknowledges the French 'Agence Nationale de la Recherche', Project Future Investments UCA JEDI, No. ANR-15-IDEX-01 (projects ImmunoMag and MagFilter) and the private company Axlepios Biomedicals for financial support,. AZ thanks the Russian Science Foundation, project 20-12-00031, for the financial support.

\section{References}

1.Creighton, Francis M. 2012."Magnetic-based systems for treating occluded vessels." U.S. Patent No. 8,308,628. 13 Nov.

2.Clements, M. J. 2016. A mathematical model for magnetically-assisted delivery of thrombolytics in occluded blood vessels for ischemic stroke treatment (Doctoral dissertation, Texas University).

3.Gabayno, J. L. F., Liu, D. W., Chang, M., \& Lin, Y. H. 2015. Controlled manipulation of Fe 3 O 4 nanoparticles in an oscillating magnetic field for fast ablation of microchannel occlusion. Nanoscale, 7(9), 3947-3953.( doi: 10.1039/x0xx00000x)

4.Li, Q., Liu, X., Chang, M., \& Lu, Z. (2018). Thrombolysis Enhancing by Magnetic Manipulation of Fe3O4 Nanoparticles. Materials, 11(11), 2313, 12p.

5. Anton Musickhin, Andrey Yu Zubarev, Maxim Raboisson-Michel, Gregory Verger-Dubois and Pavel Kuzhir, Field-induced circulation flow in magnetic fluids, Phil. Trans. R. Soc. A, 378: 20190250

6. Rosensweig, R, 1985, Ferrohydrodynamics. (Cambridge, New York)

7. Landau, L., Lifshitz, E., 1960, Electrodynamics of Continuum Media. (Pergamon Press, London)

[8] Bacri, J. C., Cebers, A. O., \& Perzynski, R. (1994). Behavior of a magnetic fluid microdrop in a rotating magnetic field. Physical review letters, 72(17), 2705.

[9] Chang, M., Chang, M. Y., Lin, W. S., \& Gabayno, J. L. (2015). Magnetic control of Fe3O4 nanomaterial for fat ablation in microchannel. Materials, 8(11), 7813-7820. 